\section{Ítalo Caorsi, una vida universitaria. 1922-2006}

\author{
MIGUEL CONCHA M. ${ }^{1, a}$
}

\section{Ítalo Caorsi, an universitary life. 1922-2006}

'Director Instituto de Anatomía, Histología y Patología, Facultad de Medicina; Universidad Austral de Chile, Valdivia, Chile.

aDoctor en Ciencias.

Declaración de Potenciales Conflictos de Intereses: Ninguno.

Recibido el 12 de diciembre de 2018, aceptado el 10 abril de 2019.

Correspondencia a:

Dr. Miguel Concha

Instituto de Anatomía, Histología y Patología, Facultad de Medicina, UACh. Cas. 567, Valdivia, Chile. migconch@uach.cl
$\mathrm{D}$ e acuerdo al Decreto Interno $\mathrm{N}^{\circ} 1$ del $1^{\circ}$ de septiembre de 1954 el Doctor Italo Caorsi Chouquer fue el primer profesor nombrado en la Universidad Austral de Chile. La sirvió ininterrumpidamente durante 49 años.

Una de las primeras preguntas que enfrenta quien indaga sobre su vida es qué impulsó a un joven médico de 32 años a formar parte de un desmesurado pero clarividente proyecto de creación de la que sería la séptima universidad del país y la primera universidad privada establecida en Chile después de 26 años. Su fundación ocurre cuando aún imperaba el Estatuto de la Enseñanza Universitaria, el cual obligaría a la nueva institución a luchar durante años por su autonomía. El propio Caorsi entregó indicios acerca de las razones que lo inspiraron a participar en el proyecto. En 1980 señaló que había tenido 'la fortuna de ver, primero como alumno y luego como ayudante docente, la transformación que se produjo en la Facultad de Medicina de la Universidad Católica en la década del cuarenta' cuando Héctor Croxatto, Luis Vargas y Joaquín Luco iniciaban una labor científica extraordinariamente exitosa. Caorsi había ganado progresivamente responsabilidades docentes en la cátedra de Histología. Entre los años 1941 y 1949 ejerce como Ayudante Ad-honorem, luego Ayudante de Planta, posteriormente es Jefe de Trabajos Prácticos y más tarde obtiene la posición de Profesor Auxiliar. Esta incipiente carrera académica no fue tomada a la ligera, al contrario, cómo reconoció posteriormente, había llegado a Valdivia enviado a cumplir el período obligatorio de post becado de la especialidad de Anatomía Patológica, compromiso que había acatado con disgusto y afligido por tener que abandonar las actividades docentes que desempeñaba en la Uni- versidad Católica junto a tan insignes maestros. Otro indicio es su percepción acerca del rol precursor que tuvo el Hospital Regional de Valdivia en la fundación de la Universidad. Explicaba que el hospital había sido creado en 1939 al amparo de nuevas concepciones que permitieron disponer por primera vez en Chile de un centro hospitalario abierto a toda la población, dotado de profesionales a jornada completa, organizado en base a las cuatro especialidades básicas de la Medicina, con uso de interconsulta y ficha clínica común. Esta concepción y la selección de un decisivo grupo médico le dieron un carácter excepcional a la medicina local. Su influencia se reflejó en la creación de la Sociedad Médica de Valdivia, la fundación de una biblioteca médica y luego los 'Archivos de la Sociedad Médica'. Caorsi indicó que 'no cabe duda que el prestigio del grupo médico que había llegado en el decenio anterior influyó [en la] efervescencia' que produjo el proyecto de fundación de la Universidad. Un tercer indicio es la juventud de los organizadores y de los intelectuales que apoyaron ésta, 'la idea loca' como la llamó Caorsi. Recordaba que pensadores de la talla de Jorge Millas, 'la honra de nuestra filosofía'; Luis Oyarzún, 'intelectual riguroso, cumbre de los intelectuales chilenos y viajero incansable'; Nicanor Parra, "rupturista, desafiante, el más internacional de todos', dictaban cursos en la Escuela de Temporada que ofrecía en Valdivia el Departamento de Extensión Cultural de la Universidad de Chile. Aunque ésta patrocinaba la creación de una sede en Valdivia, sus profesores Millas y Oyarzún entregaban un decidido apoyo a la futura Universidad. Por entonces Millas tenía 37 años, Oyarzún, 34 años y Caorsi, 32 años; Eduardo Morales, médico otorrinolaringólogo y primer Rector de la futura 


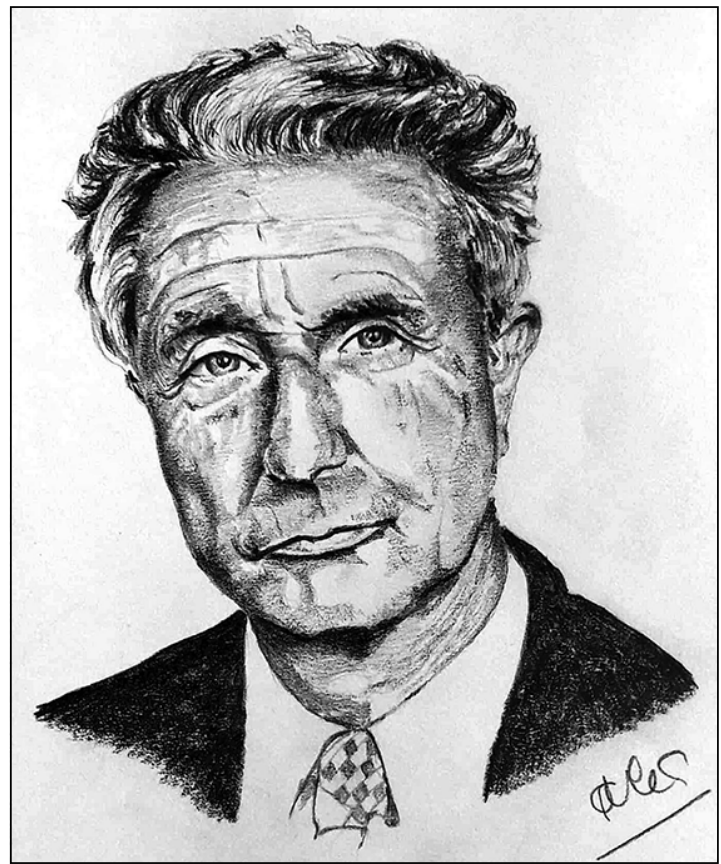

Figura 1. Dr. Italo Caorsi Chouquier [1922-2006]. Profesor de Anatomía Patológica y Catedrático Universidad Austral de Chile. La figura es el resultado de la entusiasta colaboración de varios de los discípulos del Dr. Caorsi. El excelente dibujo es obra de la Dra. Alejandra Droguett L., Unidad de Nefrología, Instituto de Medicina; el Dr. Daniel Carpio P., Director del Programa de Especialización en Anatomía Patológica, entregó la autorización para su publicación; y el Dr. Carlos Figueroa V., ex director del Instituto de Anatomía, Histología y Patología, editó la imagen digital final.

institución, era algo mayor, tenía 44 años. No es aventurado señalar que la juventud de quienes la idearon, jugó un rol en el éxito de la empresa y que en la temprana decisión de Caorsi también influyeron la seducción por la tradición académica y un ambiente profesional propicio.

Una vez ocurrida la creación de la Universidad en 1954, Caorsi se aplicó incansablemente al forjamiento institucional. Entre los años 1955 y 1968 participa en la creación y será Decano de dos Facultades, Estudios Generales y Medicina. El año 1962 además dirige por algunos meses la nueva Facultad de Ciencias Naturales y Matemáticas. Su posición en la Universidad es indudablemente central. Lidera la creación de las escuelas de Técnicos Laborantes, Enfermería, Obstetricia y de Medicina. Además dirige el Instituto [Depar- tamento] de Histología. Esta descomunal labor no estará exenta de dificultades e incluso fracasos.

La Escuela de Técnicos Laborantes nace el año 1958. Caorsi es su primer director y la fundamenta en 'la necesaria división del trabajo científico así como su proyección práctica con el fin de lograr un mayor rendimiento y rigurosidad. La nueva Escuela inicia las actividades lectivas en marzo de 1960 poco antes del terremoto del 22 de mayo. El Oficio $\mathrm{N}^{\circ} 50$ que dirige a la Secretaría General el 10 de enero de 1962 devela su estilo de trabajo y también las dificultades de gestión. Señala que el primer curso ha terminado sus dos años de ramos básicos y que a consecuencias de la destrucción del hospital las alumnas deberán continuar durante 1962 los estudios en la Universidad de Chile. Ésta ha aceptado recibirlas pero es necesario financiar las estadías, para lo cual se solicitaron becas al Servicio Nacional de Salud con compromiso de retorno a los servicios de la región. Advierte que debió renunciar a la solicitud de las becas porque el Servicio Nacional de Salud las condicionó a disminuírselas a la Universidad de Chile. Razón 'bastante antipática', según señala, que obligará a la Universidad a ayudar económicamente a las alumnas. Al pasar, comenta que la Universidad de Chile cambió la denominación de la carrera a Tecnología Médica lo que ha producido una desvinculación de programas. Nada se sabe qué fraguó en los meses siguientes pero cuatro meses más tarde el Servicio Nacional de Salud otorgó las becas, la Universidad puso cuatro becas más, la Universidad de Chile no perdió las suyas y quedaba convenido el cambio de denominación de la carrera. Tarea cumplida y de manera redonda.

La Universidad da un paso decisivo al acordar el 5 de octubre de 1959 la creación de la Facultad de Medicina. Se designa como Decano al Dr. Roberto Barahona, Profesor de Anatomía Patológica de la Universidad Católica. Se encarga a Caorsi la vinculación de los futuros alumnos con los ramos de ciencias básicas que ofrece la Facultad de Estudios Generales. La tarea se advierte compleja y bien lo perciben las autoridades. Un mes más tarde, el Vicerrector Académico, Víctor Kunstmann, envía un largo y enrevesado oficio a Juan Gómez Millas, Rector de la Universidad de Chile. El oficio hace mención a la nueva Facultad de Medicina y las asignaturas que esta ofrecerá, pero sólo en la segunda de sus tres páginas hace 
una muy acotada referencia a 'los estudios de la Escuela de Medicina'. Era la fórmula elegida para su presentación, la cual, es de imaginar, permitiría sortear el embarazoso escenario de una solicitud explícita de funcionamiento. En 1960, el Rector Gómez Millas comunica que la Asesoría Jurídica encomendada por el H. Consejo Universitario de la Universidad de Chile determinó que no se cumplían las condiciones para que conforme a la ley $\mathrm{N}^{\circ} 11.861$ los alumnos de la Escuela de Medicina de la Universidad Austral optaran al título de médico cirujano otorgado por la Universidad de Chile. En julio de 1962 el Dr. Barahona reiteró una vez más su renuncia al cargo de Decano, señalando que no estaban dadas las condiciones para gestionar un programa médico universitario. Caorsi fue nombrado en su reemplazo pero no volvería a referirse a estos sucesos.

La Escuela de Medicina fue finalmente creada el 30 de junio de 1965. Caorsi desplegó ingentes esfuerzos para organizar la nueva Escuela procurando, con invitaciones a profesores de las Universidades de Chile, Católica y de Concepción, disponer de un núcleo de académicos calificados. Se establecen comisiones asesoras, los presupuestos son reclamados sin pausa y se organizan reuniones con el Poder Ejecutivo, Legislativo y el Ministerio de Salud. Con la Universidad de Chile se crea la Comisión Coordinadora Inter Facultades que velará por el cumplimiento del Estatuto Universitario, que cual Hidra de Lerna se niega a morir. Sin embargo, el contexto político del país era otro, la gobernanza del sistema universitario chileno enfrentaba transformaciones, y la propia Universidad había crecido. El Consejo de Rectores de las Universidades Chilenas recogió el discurso pronunciado por Caorsi durante el acto de inauguración de la nueva Escuela en marzo de 1967. Su descripción del largo camino recorrido hasta ese día se aplica perfectamente a él mismo: 'constituye en sí un modelo ejemplar de acción perseverante, sustentado por elevados ideales profesionales, y por una definida orientación de desarrollo'. Se han amalgamado la institución y el hombre.

La fundación de las Escuelas de Enfermería y Obstetricia, la primera en enero de 1963 y la segunda en enero de 1965, constituyen elementos bien meditados del plan de desarrollo de la Facultad de Medicina. Caorsi las fundamenta en una vigorosa visión sobre el devenir de la salud en el país y las responsabilidades de la Universidad con la nación y los organismos internacionales de salud. Indica que el crecimiento natural de las actividades docentes de la Facultad de Medicina plantea la posibilidad de crear dichas escuelas, las que perfeccionan el grupo de carreras profesionales de colaboración médica. El discurso es inteligente, el estilo para escribir es directo y económico en palabras, se presta al reconocimiento del que disfruta. El Consejo Universitario no demoró ambas autorizaciones.

En abril de 1967 es reelegido Decano en votación efectuada por el Consejo de Facultad. Caorsi llamaría la atención más tarde que los cargos universitarios debían ser ocupados por designación de los más calificados, para lo cual debían crearse comisiones académicas de búsqueda. Esta idea nunca fue recogida por la Universidad. El nuevo período de Decano será áspero. Se suceden diferencias en el Consejo Universitario, incluso con su amigo el Rector Félix Martínez, debe intervenir en un largo y molesto sumario a un profesor, se suceden desacuerdos manifiestos con algunos de los miembros del Consejo de Facultad, y ocurre una huelga de las alumnas de Enfermería. En 1968, un año después de haber sido elegido Decano, presenta su renuncia al Rector, pero no la comunica al Consejo de Facultad. Señala que ha 'sacrificado posibilidades de perfeccionamiento profesional o docente, en la convicción de que el deber fundamental, en esta etapa de la Universidad, era servir los intereses primordiales de la Corporación'.

Efectivamente, Caorsi había sacrificado la carrera docente y de investigador para cumplir las labores directivas que había realizado hasta esa fecha. Al dejar la Decanatura, Caorsi podrá dedicar más tiempo a la Jefatura del Servicio de Anatomía Patológica del Hospital Regional, al diagnóstico histopatológico para el cual tenía particulares habilidades, a las lecciones de Patología y a la investigación científica, tarea esta última que en realidad nunca había abandonado. Entre 1950 y 1958 realizó al menos 15 publicaciones sobre casos anátomo-clínicos. El año 1964 dirigió una tesis que, adelantándose a descubrimientos posteriores, demostró el rol de la célula cebada en la iniciación de la respuesta inflamatoria. Con todo, publicó un total de 55 trabajos científicos, algunos de los cuales alcanzaron notoriedad entre sus pares internacionales.

Los años previos y posteriores a 1973 no fueron 
buenos para él. Cuando Millas, acosado por el régimen, decide en 1981 retirarse de la Universidad, Caorsi declara que 'no hay universidades, solo hay universitarios y cuando estos se alejan de ellas, aquellas dejan de serlo'. Su desesperanza ha tocado fondo.

Pero será a partir de ese período que su visión acerca del quehacer académico, la condición del profesor universitario y el papel de la investigación comiencen a dar frutos. El año 1980, en el IV Curso de Avances en Medicina, realizado en la Universidad de la Frontera, explica, que ante el arrollador impulso de la investigación científica, la universidad ha debido cambiar su estructura interna y reemplazar la cátedra por unidades académicas, constituidas por equipos jerarquizados de profesores que se organizan en base a líneas de investigación. Ésta pasa a ser el alma de la universidad, la dignidad de ella y la que presta jerarquía y ponderación al profesor universitario. Con este cambio se modifica la forma de enseñar, ésta se dirigirá hacia la participación cada vez más activa del alumno. El profesor, un investigador experimentado, fundará la formación en el resultado de sus hallazgos. Este giro obliga a que la Universidad se desarrolle hacia todas las áreas de la vida, incluidas las literarias y artísticas. Los conceptos de universidad que Caorsi examinó en esa ocasión no fueron aspiraciones excesivas. Habían sido elaborados en fecundas jornadas de trabajo con Jorge Millas, Claudio Wagner y Esteban Rodríguez. Su propio Instituto las encarnó por muchos años. Con la creación de la Escuela de Graduados y Oficinas de Graduados en cada Facultad, se había comenzado a dar forma a los programas de postgrado, primero de magíster y luego de doctorado, los que fueron decisivos para el desarrollo sistemático de la investigación. Caorsi no fue ajeno a este devenir, porque había participado decididamente en estas concepciones desde los cargos de Asesor del Honorable Senado Universitario, en el Consejo Académico y la Comisión de Doctorado.

En el ir y venir entre el Hospital Regional, la Clínica Alemana de Valdivia, el Instituto de Histología y Patología, la docencia y las invariables reuniones, Caorsi refuta a Napoleón, recomienda a los alumnos leer Cazadores de Microbios, está al tanto de la vida de Virchow, fotocopia las memorias del fisiólogo Cannon, analiza los descubrimientos de Walter Florey sobre la microcirculación, se emociona con los hallazgos de Paul Langerhans, estudia a los grandes pensadores universitarios, Jaspers, Weber y Scheler, sigue siempre a Ortega y Gasset, Simone Weil no se escapa, revisa los libritos sobre universidad que le trae Héctor Casanova y, por supuesto, lee a Millas y a los dos Oyarzún Peña, el poeta y el psiquiatra. Con su letra minúscula hace anotaciones en cuadernos, señalando 'mira, esta frase es buena, nos puede servir'.

Caorsi no fue un profesor consagrado al laboratorio experimental. Pero con su inteligencia, perspicacia, su trato sencillo y tono burlón, se tornó un inspirador de la investigación científica. Sergio Mezzano ha referido que su vocación por la Nefrología surgió estimulada por Caorsi a la luz de los cambios que en los años 80' se producían en el conocimiento de las glomerulopatías. Carlos Vío lo considera un democratizador de la investigación porque apoyaba a quienes poseían buenas ideas, aunque no tuviesen la preparación más conveniente. No es casualidad que Mezzano y Vío, al igual que Caorsi, fuesen distinguidos como Miembros Correspondientes de la Academia Chilena de Medicina. Carlos Figueroa recuerda su primera reunión de trabajo con Caorsi: 'poco trámite, algunas ideas atingentes al problema y a trabajar'.

En octubre de 1989, con motivo del Trigésimo Aniversario de la creación de la Facultad de Medicina, Caorsi repasó las ideas que venía formulando desde años anteriores. Habla sobre los cambios que la investigación científica ha provocado en la estructura de la universidad; la vocación del profesor y la función docente; el desafío de mantener la armonía entre las ciencias naturales y del espíritu; el atraso que sufren las universidades latinoamericanas. Cuando se refiere a la Universidad de Berlín conoce muy bien qué está describiendo. La Universidad de Berlín se impone como una universidad paradigmática porque no deja de enseñar sobre el hiato entre realidad e ideal. La Universidad de Berlín es fundada bajo pensamientos notables como 'la universidad no se limitará al rol de trasmisora del saber puesto que desde el punto de vista de la investigación científica el saber jamás se alcanza si no que resta como una pregunta abierta sin limites' o 'el profesor universitario no es más un profesor y el estudiante no es alguien meramente enganchado en el proceso de aprendizaje si no una persona que asume su propia investigación, mientras el profesor lo dirige y apoya'. Tras largos años de evolución, 
este nuevo enfoque daría origen a las universidades de clase mundial que han hecho de los doctorados en filosofía (PhD), ofrecidos en todas las áreas del saber, con esa u otras denominaciones, el grado de investigación por excelencia. Las ideas de Caorsi sobre universidad han alcanzado lucidez indisputable, tienen fundamentos universales $y$, como lo indica al finalizar el discurso, 'son fuente de optimismo'.

Los últimos años acumularon aflicciones que lo fueron alejando de la vida universitaria. La enfermedad y muerte de su esposa, Sra. Carmen Saelzer, la derrota en las elecciones de Rector del año 90, no deben haber sido fáciles de aceptar. Tampoco la negativa acogida que recibió su propuesta de convertir a la Clínica Alemana de Valdivia en un hospital universitario. Era la segunda vez que siendo las condiciones completamente favorables la Universidad Austral rechazaba una obra de esa repercusión. La posterior eliminación de la asignatura de Patología General, que pasó a ser parte de un ramo integrado, mostraba que los tiempos cambiaban y él no estaba dispuesto a ser parte de esos cambios.

Al mirar hacia atrás resta una lista impresionante de cargos, comisiones y reconocimientos recibidos. Caorsi fue Presidente de la Sociedad Médica de Valdivia; Director de la Revista Archivos de la Sociedad Médica de Valdivia; miembro de la Sociedad Chilena de Anatomía Patológica; miembro de la Sociedad Chilena de Citopatología; representante de Chile en el Directorio de la Sociedad Latinoamericana de Anatomía Patoló- gica; Consejero de la Revista Latinoamericana de Patología; premio al artículo del año del Journal of Utrastructural Pathology; fundador de la Asociación Gremial de Académicos de la Universidad Austral de Chile; Catedrático de la Universidad Austral de Chile; Miembro Correspondiente de la Academia Chilena de Medicina; medalla Orden Cruz del Sur en el Grado Gran Cruz del Ministerio de Salud; y Premio Jorge Millas otorgado por la Universidad Austral de Chile.

Caorsi demostró ser un hombre de visión, persistencia e integridad, cualidades que le facilitaron la acogida de sus ideas. Su influencia se extendió desde la gestión académica a la patología diagnóstica y experimental; desde la educación universitaria a la investigación. Dejó la huella de sus virtudes en todos los cuerpos universitarios y profesionales donde participó, en el espíritu de sus colaboradores, la sucesión de jóvenes que formó y de los amigos que hizo.

Agradecimientos: El autor desea agradecer a la Sra. Ma. Asunción de la Barra S., Secretaria General de la Universidad Austral de Chile, que autorizó la revisión de los Archivos Históricos de la Universidad de los cuales se extrajo gran parte de la información utilizada en el presente trabajo. Asimismo, a los profesores Drs. Alejandra Droguett L., Daniel Carpio P., y Carlos Figueroa V. por el generoso aporte que cada uno de ellos hizo en la preparación de la Figura 1. A la Dra. Alejandra Vidal V. por la lectura crítica del artículo y las sugerencias que propuso. 